\title{
The Asymptomatic Patient with Atrial Fibrillation: An Arrhythmia in Search of a Symptom, previously for some but now for many.
}

\author{
Robert Lemery ${ }^{1}$ \\ ${ }^{1}$ Arizona Heart Rhythm Center and St-Joseph Hospital
}

February 7, 2022

\begin{abstract}
Asymptomatic patients with atrial fibrillation were found to have different echocardiographic and hemodynamic measurements than symptomatic patients. The advent of new digital technology to diagnose atrial fibrillation has revealed a greater number of patients who are asymptomatic.
\end{abstract}

The Asymptomatic Patient with Atrial Fibrillation: An Arrhythmia in

Search of a Symptom, previously for some but now for many

Robert Lemery MD, MA

From the AZ Heart Rhythm Center and St-Joseph Hospital, Dignity Health, Phoenix, Arizona.

Address for correspondence: robert.lemery1@gmail.com

The author reports no funding or conflict of interest.

Word count: 1,500

Why don't all patients with atrial fibrillation (AF) have symptoms? In this issue of the Journal, Fukuhara et al (1) report on 110 patients with paroxysmal AF who underwent invasive hemodynamic measurements at the time of catheter ablation. Asymptomatic patients with AF, as defined by an EHRA score of 1 (no symptoms) for AF related symptoms (2), was documented in 19 patients. Catheter ablation involved either a Tacticath catheter and use of the Verismo software, or Cryoballoon ablation with a $28 \mathrm{~mm}$ balloon. Following catheter ablation, transseptal measurements of left atrial pressure and arterial pressure were obtained in sinus rhythm as well as during high right atrial pacing from the lateral wall of the RA using a multipolar catheter inserted from the right internal jugular vein. The pressures were obtained through the sheath, which was connected to a pressure transducer and recorded on a Fukuda Denshi hemodynamic monitoring system (FCL-2000). The average of three heartbeats at the end of expiration was used to measure LA pressure and arterial pressure.

Following measurements in sinus rhythm, the pacing protocol consisted of pacing at $100 \mathrm{beat} / \mathrm{min}$, increased by 10 beats/min until Wenckebach block at higher than 120 beats/min. Fukuraha et al (1) demonstrated that the peak LA pressure and the rate of change of pressure measurements with pacing were significantly less in asymptomatic patients with AF. In addition, those patients also had significantly lower mean arterial pressure during sinus rhythm, as well as significantly lower peak arterial pressure during sinus rhythm or with atrial pacing. The multivariate analysis revealed that asymptomatic patients with AF had significantly lower rates of change of peak LA pressure in SR or while pacing at $100 \mathrm{bpm}$, and a lower E/e on echo, suggestive of less diastolic dysfunction (3). 
The etiology of symptoms in patients with AF has generally been attributed to the irregularity of AVN conduction in AF, rapid ventricular rates during AF, autonomic effects, loss of AV synchrony and decreased cardiac output with lack of left atrial appendage contraction, and perhaps more importantly to uncovering underlying diastolic dysfunction $(4,5)$. As clinicians, we have all been baffled by patients with $\mathrm{AF}$ who have symptoms that appear out of proportion to the burden or perceived severity of AF. Patients with unrelenting and persistent $\mathrm{AF}$ with rapid ventricular rates may not be aware of their $\mathrm{AF}$, and conversely patients with infrequent paroxysmal AF may be extremely symptomatic at the time of recurrence of AF. The report by Fukuhara et al (1) suggests that asymptomatic patients with AF are thus characterized by preserved diastolic function, with significantly less increase in peak LA pressure during AF, unrelated to ventricular rates.

There have been several reports (8-11) highlighting the absence of symptoms in subgroups of patients with AF. In previous studies of patients with persistent AF (9), or both paroxysmal and persistent AF (10), ischemic thromboembolic complications were not significantly different between patients with or without AF symptomatology. As observed by Fukuhara et al (1), studies of asymptomatic patients with AF have generally been shown to include significantly more males or patients with diabetes (8-11). Overall, approximately onefourth of patients with AF were previously estimated to be asymptomatic. But with changing definitions and new means of monitoring AF, significant clinical readjustments in patient management are emerging. Subclinical AF, in the new era of increased use of portable and implantable devices, has shown up to $60 \%$ of asymptomatic episodes over 5 years (12-14).

The percentage of patients with asymptomatic AF in the report by Fukuhara et al (1) was only 17\%, likely related to the patient population studied, which consisted of patients undergoing catheter ablation. In asymptomatic patients with AF, catheter ablation has been considered a class IIb indication (15). The justifications for performing AF ablation in the asymptomatic patients with AF by Fukuhara et al (1) were not unlike the usual reasons most interventional electrophysiologists currently perform ablation in patients with symptomatic AF. These include patient preference of AF ablation over long-term treatment with AVN blocking or antiarrhythmic agents, concerns of new onset or worsening of systolic or diastolic heart failure, patient hope of being able to stop anticoagulation with maintenance of sinus rhythm, and the risk of bleeding complications with use of anticoagulants. Clinically, these are all strong deterrents towards a laisser-faire attitude in the asymptomatic patient.

Nonetheless, after more than twenty years of worldwide experience at performing AF ablation, the clinical outcome of AF ablation remains a work in progress. Recurrence of AF post ablation or various ablation related complications are still somewhat expected or accepted in the symptomatic patient with AF. Arguably however, it is more difficult to accept an unfavorable outcome following AF ablation in the asymptomatic patient. While evidence-based reports are lacking for performing AF ablation in such patients (15). percutaneous occlusion of the left atrial appendage has rapidly emerged as a safe and effective interventional procedure to supplant long-term use of anticoagulants.

Randomized trials comparing the clinical outcome of patients with subclinical AF treated with Apixaban vs. Aspirin (16) or Edoxaban vs. Aspirin or placebo (17) will be available over the next year. Numerous randomized trials comparing percutaneous occlusion of the left atrial appendage with direct oral anticoagulants will also become available over the next few years (18). Percutaneous occlusion of the left atrial appendage could emerge as the treatment of choice for subgroups of asymptomatic patients with AF. While we need to continue to research the pathophysiology of why an irregularly irregular heart rhythm may not be perceived clinically, and adjust our monitoring tools accordingly, as interventional electrophysiologists we are confronted with an arrhythmia that has been and remains a lesson in humility.

\section{References}

1. Fukuhara E. Mine T., Kishima H, Kitagaki R, Ishihara M. Increase in Heart Rate-dependent Left Atrial Pressure is Associated with Symptoms in Patients with Paroxysmal Atrial Fibrillation. J. Cardiovasc 
Electrophysiol 2022 (In press)

2. Wynn GJ, Todd DM, Webber M, Bonnett L, McShane J, Kirchhof P, Gupta D. The European Heart Rhythm Association symptom classification for atrial fibrillation: validation and improvement through a simple modification. Europace. 2014; 16:965-72.

3. Mitter SS, Shah SJ, Thomas JD. A test in context: E/A and E/e' to assess diastolic dysfunction and LV filling pressure. J Am Coll of Cardiol. 2017; 69: 1451-64.

4. Rienstra M, Lubitz SA, Mahida S, Magnani JW, Fontes JD, Sinner MF, Van Gelder IC, Ellinor PT, Benjamin EJ. Symptoms and functional status of patients with atrial fibrillation: state of the art and future research opportunities. Circulation. 2012; 125:2933-2943.

5. van den Berg MP, Hassink RJ, Tuinenburg AE, van Sonderen EF, Lefrandt JD, de Kam PJ, van Gelder IC, Smit AJ, Sanderman R, Crijns HJ. Quality of life in patients with paroxysmal atrial fibrillation and its predictors: importance of the autonomic nervous system. Eur Heart J. 2001; 22:247-253.

8. Rho RW, Page RL. Asymptomatic atrial fibrillation. Progress in cardiovascular diseases. 2005; 48:79-87.

9. Rienstra M, Vermond RA, Crijns HJ, Tijssen JG, Van Gelder IC, RACE Investigators. Asymptomatic persistent atrial fibrillation and outcome: results of the RACE study. Heart Rhythm. 2014; 11:939-945.

10. Gibbs H, Freedman B, Rosenqvist M, Virdone S, Al Mahmeed W, Ambrosio G, Camm AJ, Jacobson B, Jerjes-Sanchez C, Kayani G, Oto A. Clinical outcomes in asymptomatic and symptomatic atrial fibrillation presentations in GARFIELD-AF: implications for AF screening. The American Journal of Medicine. 2021; 134:893-901.

11. Bano A, Rodondi N, Beer JH, Moschovitis G, Kobza R, Aeschbacher S, Baretella O, Muka T, Stettler C, Franco OH, Conte G. Association of Diabetes With Atrial Fibrillation Phenotype and Cardiac and Neurological Comorbidities: Insights From the Swiss-AF Study. J Am Heart Assoc 2021; 10: e021800.

12. Noseworthy, P.A., Kaufman, E.S., Chen, L.Y., Chung, M.K., Elkind, M.S., Joglar, J.A., Leal, M.A., McCabe, P.J., Pokorney, S.D., Yao. Subclinical and device-detected atrial fibrillation: pondering the knowledge gap: a scientific statement from the American Heart Association. Circulation 2019; 140: e944-e963.

13. Quer G, Freedman B, Steinhubl SR. Screening for atrial fibrillation: predicted sensitivity of short, intermittent electrocardiogram recordings in an asymptomatic at-risk population. Europace. 2020; 22:17811787 .

14. Kashou AH, Adedinsewo DA, Noseworthy PA. Subclinical Atrial Fibrillation: A Silent Threat with Uncertain Implications. Annu Rev Med 2022; 73: 355-362.

15. Calkins H, Hindricks G, Cappato R, Kim YH, Saad EB, Aguinaga L, Akar JG, Badhwar V, Brugada J, Camm J, Chen PS. 2017 HRS/EHRA/ECAS/APHRS/SOLAECE expert consensus statement on catheter and surgical ablation of atrial fibrillation. 2017; 14: e275-e433

16. ClinicaltTrials.gov Identifier: NCT01938248. The Apixaban for the Reduction of Thrombo-Embolism in Patients with Device-Detected Sub-Clinical Atrial Fibrillation (ARTESIA) trial

17. ClinicaltTrials.gov Identifier: NCT02618577. Non-vitamin K Antagonist Oral Anticoagulants with Atrial High-Rate Episodes (NOAH).

18. Ding WY, Mandrola J, Gupta D. Left atrial appendage occlusion: past, present and future. Thrombosis and Haemostasis. 2020; 120:1484-1491. 